\title{
Mammalian genes interacting
}

RNA interference (RNAi)-based genetic interaction screens in mammalian cells show how genes affect each other.

In genetics, the total is not always the sum of its parts. Sometimes the joint effort of two genes creates a phenotype that is different from the one expected from simply adding the effects of the two genes. Genetic interactions, first systematically explored in yeast, were recently studied in mammalian cells in independent work by the groups of Jonathan Weissman and Nevan Krogan, both at the University of California in San Francisco.

Genetic-interaction maps in yeast enable the identification of positive and negative interactions between genes, and the grouping of genes that function in the same protein complexes or pathways. "The value you could extract from [genetic-interactions maps in yeast] induced us and others to think that they could be useful in higher organisms," recalls Krogan. Michael Bassik-a post-doc in the Weissman laboratory who, together with Martin Kampmann, led their study-sums up their motivation: "we wanted to be able to look at disease models where synthetic lethal interactions will have an enormous application for the identification of cancer drug targets."

For mammalian cells, however, genomewide deletion strains, as used to map genetic interactions in yeast, are not available, so the researchers used two different RNAi strategies for pair-wise gene knockdown.

Bassik and Kampmann developed a pooling approach to overcome the problems inherent in any RNAi screen, that is, direct off-target effects (an unintended target is knocked down) and indirect effects (the cellular RNAi machinery is saturated). They applied this procedure to find genes whose interaction affects the response of human cells to the toxin ricin.

Starting with a primary genome-wide RNAi screen, they targeted every proteincoding gene with $\sim 25$ different short hairpin RNAs (shRNAs), infected human leukemia cells, and monitored the enrichment of shRNAs in pools of cells with and without

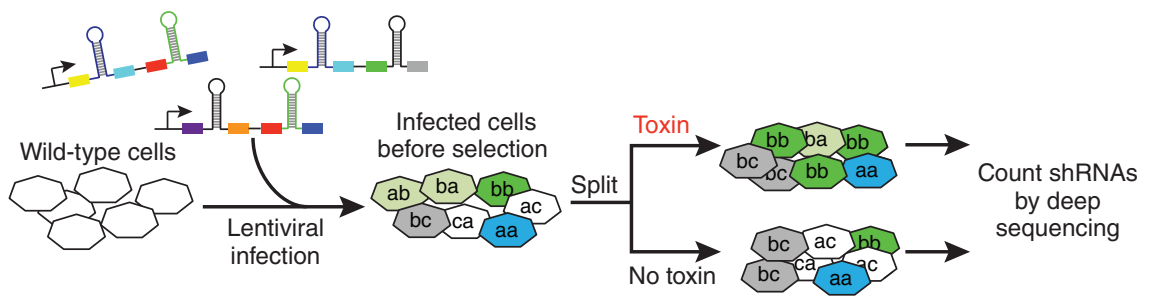

Schematic of the pooled shRNA strategy. shRNAs are split into pools $a, b$ and c, and randomly ligated into pairs. Reprinted with permission from Cell.

ricin treatment by deep sequencing. The high-coverage library maximizes the chance to effectively target each gene with multiple independent shRNAs, and thus allows rigorous evaluation of the probability that shRNAs act on the intended target. This screen yielded $\sim 200$ genes affecting a cell's susceptibility to ricin. The team then barcoded and ligated pairs of shRNAs to these genes and repeated the screen. Computational deconvolution allowed them to identify genetic interactions that deviated from the expected phenotypes. The resulting genetic-interaction map recapitulated well-characterized complexes but also highlighted unexpected results.

This pooling strategy has several advantages in addition to controlling for off-target effects. "We did not need high-throughput robotics," says Kampmann; "anybody with a cell culture room can implement our method." One also does not need to be concerned about batch or positioning effects like in wellbased assays, and the amount of the pooled cells is easily scalable; furthermore, the use of a virus to infect cells with shRNAs does not limit the screen to easily transfectable cell lines. The limitation is that phenotypes need to be assessed in pooled populations, and one cannot look at more subtle changes in individual cells that would require a microscope.

To monitor more phenotypic readouts, Krogan's team, in collaboration with the groups of Barbara Panning and Sourav Bandyopadhyay, used a different approach to screen each gene pair in a separate well by high-content imaging. The scientists used endonuclease-prepared siRNAs (esiRNAs), which are generated via cleavage of double-stranded mRNA into multiple small interfering RNAs. This reduces the chance of off-target effects but requires robotic liquid handlers to dispense two esiRNAs in each well. The researchers reverse-transfected mouse cells and monitored the effect on cell growth with imaging. They targeted 130 genes that are known to be involved in the regulation of chromatin and scored each genetic interaction pair as positive, negative or neutral. Krogan sums up the results, "we were happy to see that the genetic trends that we saw in simple organisms also exist in higher organisms, including the fact that positive genetic interactions correlate with protein-protein interactions."

For both teams, the investigation of epistasis in disease pathways is a high priority. Whereas Weissman's team wants to study cancer cells, Krogan's group is interested in analyzing host-pathogen interactions, where the ability to have multiphenotypic readouts is key, as well as higher-order interactions involving three genes. In addition, Krogan and colleagues are working with an esiRNA library targeting noncoding RNAs to probe how these regulatory RNAs interact with their protein-coding counterparts.

\section{Nicole Rusk}

\section{RESEARCH PAPERS}

Bassik, M.C. et al. A systematic mammalian genetic interaction map reveals pathways underlying ricin susceptibility. Cell 152, 909-922 (2013).

Roguev, A. et al. Quantitative genetic-interaction mapping in mammalian cells. Nat. Methods advance online publication (13 February 2013). 